Patient 2 was a 72 year old man who had been admitted 10 times in the previous two years for alcohol detoxification. He had had untreated syphilis during the second world war. In 1966 he had been treated for dementia paralytica twice with penicillinbismuth and once with chlortetracyclin. At that time he had recovered clinically but he did not become seronegative. In 1968 treatment had therefore been repeated. A decision to reinstate treatment had been taken in 1982, not because of any recurring symptoms or signs, but because of changing opinions about the adequacy of treatment. Treatment then consisted of benzathine benzylpenicillin $(2.4 \mathrm{MU}$ once a week for six weeks). He was already misusing alcohol. On one admission to our hospital for detoxification psychological testing showed only slight impairment of cognitive functions. One year later he was readmitted and found to be demented. His memory was seriously impaired, affect was shallow, and there was loss of decorum and social ability. The Korsakoff syndrome and primary degenerative dementia were considered as the most likely diagnoses. As both serum and CSF were VDRL negative and only weakly positive in Treponema pallidum haemagglutination (TPHA) and fluorescent treponemal antibody absorption (FTA-abs) tests, reactivation of neurosyphilis seemed unlikely. Because of our experience with patient A, however, patient $B$ was also treated with ceftriaxone. Cognitive functions recovered to normal.

Both patients had had neurosyphilis and had been treated with penicillin intramuscularly. After initial recovery this treatment was considered sufficient; intravenous treatment with high dose penicillin for neurosyphilis is nowadays advocated to supply a continuous treponemicidal concentration in CSF. ${ }^{4}$ In earlier years many patients received intramuscular penicillin. Serological tests had not shown relapse of neurosyphilis in our patients, but it should be borne in mind that the clinical significance of VDRL seroconversion after treatment is unknown, and that serological tests are often overvalued in determining the response to treatment of neurosyphilis. ${ }^{5}$ The TPHA and FTA-abs are highly specific and sensitive for Treponema pallidum infection. These tests do not, however, give any information on clinical activity or time of infection, and are only useful in excluding the diagnosis of syphilis. VDRL tests are neither specific nor sensitive. Only strongly positive responses $(<1: 16)$ signify active infection. It is therefore extremely difficult to prove whether neurosyphilis is active or not after an initial infection. In fact, the only argument that dementia was due to neurosyphilis in these two cases was the dramatic clinical improvement after treatment with ceftriaxone.

These cases draw attention to neurosyphilis as a differential diagnosis of (Wernicke-)Korsakoff's syndrome and as a possible cause of dementia in alcoholic patients. Both patients seemed to have been treated adequately with penicillin, but became demented after some time. In such cases treatment with ceftriaxone may lead to full recovery.

W M CNOSSEN H NIEKUS

M NIELSEN B A BLANSJAAR

St foris Gasthuis, St forisweg 2, 2612 GA Delft,
Correspondence to: Dr B A Blansjaar.

1 Luteyn F, van der Ploeg FAF. Groninger Intelligentietest. Lisse: Swets en Zeitlinger BV, 1983.

2 Folstein MF, Folstein SE, McHugh PR. "Mini-mental state", a practical guide for grading the cognitive state of patients for the clinician. $\mathcal{F}$ Psychiatric Res 1975;12:189-98.

3 Deelman BG, Saam RJ, van Zomeren AH. Traumatic brain injury. Clinical, social and rehabilitational aspects. Amsterdam: Swets en Zeitlinger BV, 1990:49-77.

4 Schoth PEM, Wolters ECh. Penicillin concentrations in serum and CSF during high dose intravenous tor Neurology 1987;37:1214-6.

5 Fiumara NJ. Treatment of primary and secondary syphilis: serologic response. fournal of the American Academy of Dermatology 1986;14:487-91.

\section{Absence of REM sleep and altered non- REM sleep caused by a haematoma in the pontine tegmentum}

The pronounced reduction or absence of REM sleep in patients with lesions of the pontine tegmentum indicates that this structure is implicated in generation of REM sleep in humans. This conclusion is based, however, on reports of a few patients with relatively large brainstem lesions. ${ }^{1-3}$ Furthermore, information on the role of REM sleep in behaviour and memory consolidation is lacking.

Our patient had no REM sleep and abnormal Non-REM sleep caused by a haematoma confined to the tegmentum of the upper pons.

A 69 year old man had sudden instability and difficulty in speaking. He had a four year history of hypertension secondary to chronic renal failure. There was no history of alcohol consumption, sleep disturbances, or psychopathology.

On admission, neurological examination showed bilateral normoreactive miotic pupils, bilateral peripheral facial palsy, supranuclear horizontal gaze palsy, and cerebellar ataxia. He received nifedipine (60 mg daily) and three haemodialysis sessions weekly. After the stroke, the patient complained of persistent insomnia. No psychotropic, hypnotic, or sedative drugs were given.

Cranial MRI seven days after the first symptom showed mild cortical atrophy and a hyperintense signal in the upper pontine tegmentum in $\mathrm{T} 1$ and $\mathrm{T} 2$ weighted images (fig 1). Three polysomnograms (PSGs) were recorded. The first was over eight hours on the night of the 15 th inpatient day. The second PSG was over 24 hours on the 30th inpatient day and the third four months later. The first and third PSGs were recorded in the sleep laboratory. The 24 hour PSG was recorded with an Oxford Ambulatory System at the inpatient's bed to avoid interference from laboratory conditions.

Parameters of PSG recorded were EEG (C3-A2), EMG, right and left electrooculogram (R-EOG, L-EOG), left leg movements (LM), body position (BP), respiratory movements (RESP), and EEG (Fp1-C3, C3-T3, T3-O1, Fp2-C4, C4-T4, T4-O2).

Electrodes to record eye movements were placed $1 \mathrm{~cm}$ lateral and $1 \mathrm{~cm}$ above the outer canthus of each eye. Both electrodes were referred to the right ear.

No psychotropic, hypnotic, or sedative

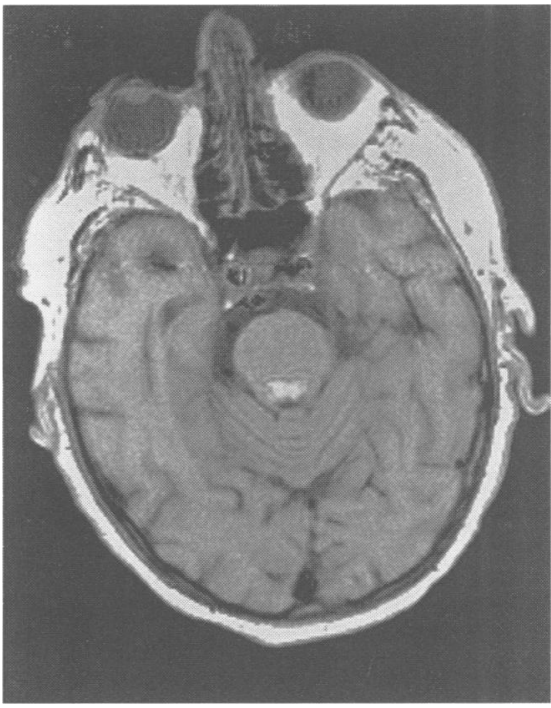

Figure 1 Increased signal on $T 1$ weighted MRI (haematoma) in the pontine tegmentum.

drugs were given on the days before or during PSG recording days.

Three different PSG patterns were recorded: (a) wake time with occipital alpha rhythm and a few vertical eye movements; (b) a decrease of over $50 \%$ in alpha rhythm and moderate decrease of EMG activity; $(c)$ a slow wave pattern with more than $20 \%$ delta activity and a greater decrease of EMG activity. (fig 2).

The first PSG recording, performed on the 15th inpatient night, showed severe insomnia with absence of REM sleep, absence of beta spindles and $\mathrm{K}$ complexes, and scarcity of deep slow sleep. There was a pronounced decrease in the total sleep time (TST) of three hours and 56 minutes. A 24 hour PSG performed 15 days later showed the same characteristics and TST was three hours and 51 minutes (fig 2). The most recent PSG, recorded four months later, showed persistence of the same sleep disorder.

Neurological examination during the three PSG sessions remained unchanged. After 18 months of follow up, mild insomnia (four to five hours of sleep nightly), supranuclear horizontal gaze palsy, bilateral peripheral facial palsy, and instability persisted.

During the follow up period, no psychic disturbances were found. The neuropsychological studies during this time (one month, four months, 12 months, and 18 months), including auditory verbal learning test (Rey), long term retention memory test, President's test, and memory span for digits (WAIS), showed no learning disability and remote, recent, and immediate memories were normal.

Previous cases of absence of REM sleep in humans have been associated with large lesions in the pons with bilateral damage of the tegmentum, mostly in patients with pronounced neurological deficits. ${ }^{1-3}$ The well delineated lesion in our patient confirms that REM sleep features originate in the pontine tegmentum. In agreement with other reports the coexistence of severe insomnia and supranuclear horizontal gaze palsy suggests that REM sleep features generate in areas closely related to control of horizontal eye movements. ${ }^{13}$ 


C3-A2
EMG
REOG
LEOG
EKG
LM
BP
RESP
Fp1-C3
C3-T3
T3-01
Fp2-C4
C4-T4
T4-02

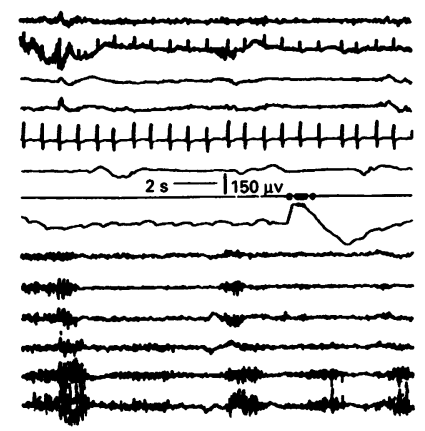

A

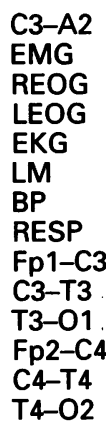

EMG

REOG

EKG

LM

RESP

Fp1-C3

C3-T3

$\mathrm{Fp} 2-\mathrm{C} 4$

T4-O2

B

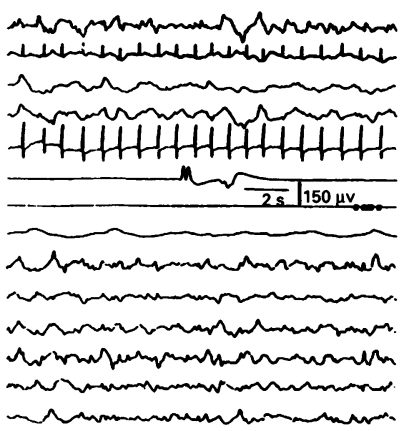

C3-A2

EMG

REOG

LEOG

EKG

LM

BP

RESP

$\mathrm{Fp} 1-\mathrm{C} 3$

C3-T3

T3-01

$\mathrm{Fp} 2-\mathrm{C} 4$

C4-T4

T4-O2

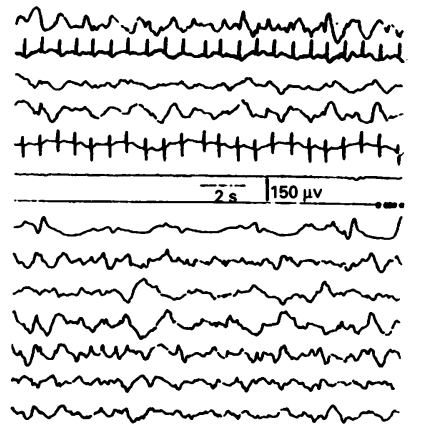

C

Polysomnogram patterns

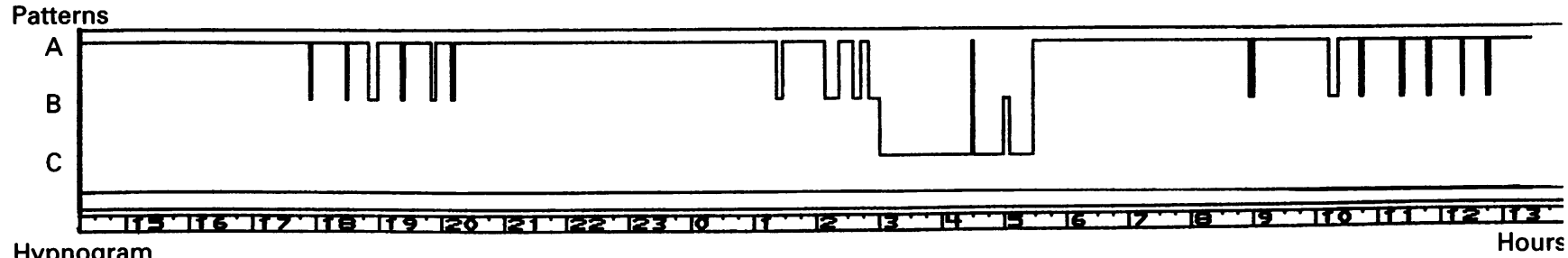
Hypnogram

Figure 2 Top: PSG patterns: (A) Wake time. (B) A 50\% or more decrease of alpha activity and moderate decrease of EMG activity. (C) Delta activity more than $20 \%$ with greater decrease of EMG activity. Bottom: hypnogram of the PSG study corresponding to 23 hour sleep period. During the night, from 2300 to 0700 , the percentage of different stages was: stage A: $62 \cdot 4 \%$, stage $B: 9 \cdot 2 \%$, and stage C: $28.4 \%$. During the day the percentage of different stages was: stage $A$ : $94 \cdot 9 \%$ and stage $B: 5 \cdot 1 \%$

Another finding in our patient was the absence of sleep spindles. As emphasised in previous reports, lesions of the pontine tegmentum may involve the peduncle-pontine ascending connections of the nucleus reticularis thalami and provoke the absence of beta spindles and $\mathrm{K}$ complexes. ${ }^{1-3}$

It is important to perform sleep recording over at least 24 hours to determine the exact sleep time and to rule out disorders of circadian rhythm, especially in patients in hospital with severe physical disability. ${ }^{1}$ In our patient, the circadian rhythm was stable with predominance of wake time during the day and sleep periods during the night, even in the inpatient period.

The psychic effects of REM sleep deprivation are not well known, partly because it is extremely difficult to produce long periods of complete REM deprivation in humans. It has been suspected that REM sleep is important for normal behaviour and that severe REM deprivation might cause irritability, hallucinations, delusions, obsessions, and schizophrenia. ${ }^{4}$ This conclusion is not supported by recent studies ${ }^{2}$ or our case.

Finally, the hypothesis that REM sleep is essential for memory consolidation is also of interest. ${ }^{5}$ To our knowledge, this is the first case of total absence of REM sleep in which neuropsychological studies have been performed, and there were no abnormal memory consequences.

This work was presented in abstract form to the XIV Annual Meeting of the Spanish Neurological Society and to the 12th Congress of the European Sleep Research Society.

$$
\begin{array}{r}
\text { ALEXANDRE GIRONELL } \\
\text { Department of Neurology, } \\
\text { Hospital de la Santa Creu i Sant Pau, Barcelona } \\
\text { M DOLORES DE LA CALZADA, } \\
\text { TERESA SAGALES } \\
\text { Sleep Unit, Department of Neurophysiology, } \\
\text { Hospital General Vall d'Hebron, } \\
\text { Barcelona }
\end{array}
$$

LLUIS BARRAQUER-BORDAS Department of Neurology, Hospital de la Santa Creu $i$ Sant Pau, Autonomous University of Barcelona,
Barcelona, Catalonia, Spain

Correspondence to: $\operatorname{Dr} A$ Gironell, Department of Neurology, Hospital de la Santa Creu i San $\mathrm{Pau}, \mathrm{Av}$ Sant Antoni $\mathrm{M}^{\mathrm{*}}$ Claret, 167, 08025 Barcelona, Catalonia, Spain.

1 Autret A, Laffont F, Toffol B, Cathala HP. A syndrome of REM and non-REM sleep reduction and lateral gaze paresis after medial tegmental pontine stroke. Arch Neurol 1989;45:1236-42.

2 Osorio I, Daroff RB. Absence of REM sleep and altered NREM sleep in patients with spinocerebellar degeneration and slow saccades. Ann Neurol 1980;7:277-80.

3 Valldeoriola F, Santamaria J, Graus F, Tolosa E. Absence of REM sleep, altered NREM sleep and supranuclear horizontal gaze palsy caused by a lesion of the pontine tegmentum. Sleep 1993;16:184-8.

4 tum. Sleep $1993,16: 184-8$. Science 1960;131:1705-7.

5 Crick FHC, Mitchison G. The function of dream sleep. Nature 1983;304:111-4.
Recurrence of cluster headaches presenting with a virtually painless Horner's syndrome

Throughout every winter from the ages of 25 to 33 a 56 year old property developer had had monthly bouts of daily pain, each bout lasting one to two weeks. During these he would experience up to five extremely severe headaches each evening between $600 \mathrm{pm}$ and $700 \mathrm{am}$, of which two or three would wake him from sleep. The pain would last 45 minutes, was situated above and around the right eye, and was associated with nasal stuffiness and watering of the eye. These pains had remained in remission for 23 years.
He was then referred urgently because of the development of a right Horner's syndrome associated with some burning over and around the right eye. Nine days previously he had had some influenza-like symptoms, waking the next morning with vomiting and vertigo. This soon settled, although he continued to experience transient vertigo when he lay with his left ear downwards. When first seen he had a right Horner's syndrome with no demonstrable sensory loss or other neurological signs. A carotid Doppler study and CT and MRI were normal.

On the evening after his CT the pains restarted, following exactly the same pattern as before. The Horner's syndrome had been resolving but his wife reported that it became more prominent in the attacks of pain. When seen three weeks later he was given prednisolone (40 mg daily) for a week, declining over the second week, and his headaches resolved within three days.

Miosis is a common feature of attacks of cluster headaches, and can often persist after the bout is over, and occasionally permanently. ${ }^{12}$ It is unusual for a patient to develop such an unequivocal Horner's syndrome with only minimal facial burning discomfort. The vomiting and vertigo, both spontaneous and positional, were considered suggestive of a viral vestibular neuronitis, particularly as no radiological evidence of a brain stem infarct could be obtained. 